\title{
The role of ion and electron electrostatic turbulence in characterizing stationary particle transport in the core of Tokamak plasmas
}

\author{
E. Fable, C. Angioni ${ }^{1}$ and O. Sauter \\ Centre de Recherches en Physique des Plasmas \\ Association EURATOM - Confédération Suisse \\ Ecole Polytechnique Fédérale de Lausanne (EPFL) \\ CH-1015 Lausanne, Switzerland \\ ${ }^{1}$ Max-Planck-Institut für Plasmaphysik, EURATOM Association, 85748 Garching, \\ Germany \\ E-mail: emiliano.fable@epfl.ch
}

\begin{abstract}
A general feature of particle transport in the core of Tokamak plasmas is that, when core particle sources are small, a stationary peaked density profile is provided by a balance of outward diffusion and inward convection, driven by either neoclassical or turbulent mechanisms. The turbulent contribution to the off-diagonal elements of the transport matrix is very sensitive on the type of dominant instability of the background turbulence. We present here a detailed quasilinear gyrokinetic analysis of stationary turbulent particle transport by means of analytical and numerical calculations to show how the actual parametric dependence of the stationary normalized density gradient can strongly vary between an Ion Temperature Gradient (ITG) dominated turbulence and a Trapped Electron Mode (TEM) dominated turbulence regime. It is also shown how the maximal achievable normalized density gradient is reached when the turbulence regime is in a mixed state. This result is interpreted as the interplay of different physical mechanisms arising from (linear) wave-particle resonances. The results presented here are addressed to interpret some of the still unresolved issue in interpreting known experimental results.
\end{abstract}

\section{Introduction}

The prediction of the stationary density profile in the core of Tokamak plasmas relies on the modeling of turbulent transport as it is generally shown to be the dominant source of energy and particle transport. It is important to understand the physical mechanisms that, through the correlation between turbulent fields, contribute to the offdiagonal elements of the transport matrix, providing a net 'convective' term that, when directed inwards, can balance the outward diffusive term to establish a peaked density profile despite the absence of core sources and the smallness of neoclassical transport $[1,2,3,4]$. Many experimental observations on stationary particle transport like magnetic shear dependence $[5,6,1,7]$, collisionality dependence $[8,7,9,10,11,12,13]$, 
temperature ratio dependence $[1,14,15]$, and density-temperature scalings in electron Internal Transport Barriers [16] in MHD-free regions of the plasma core have provided a rich phenomenology on which theoretical models can be tested both qualitatively and ultimately quantitatively. Indeed first-principles based models, either fully nonlinear $[17,18,19]$ or with quasi-linear approximation $[9,20]$ have found qualitative agreement with some of the experimental trends. These and other theoretical works have disclosed the basic mechanisms that drive the off-diagonal particle transport terms $[21,22,23,24,25,26,27,28]$. We shall briefly summarize the previous results in a particular fashion, to put in evidence how all the linear wave-particle resonance physics ultimately leads to the known theoretical results. We show with several specific parameter scans how quasi-linear numerical results can provide a useful insight to some of the unresolved experimental issues. In particular our results can explain why the dependence of the observed density peaking on plasma parameters is very different depending on the turbulent regime (e.g. collisionality dependence of $\mathrm{H}$-modes with $T_{\mathrm{e}} / T_{\mathrm{i}} \approx 1[7]$ or magnetic shear dependence of $\mathrm{L}$-modes with $\left.T_{\mathrm{e}} / T_{\mathrm{i}} \gg 1[1,7]\right)$.

The organization of the paper is as follows: in Section 2 we present the theoretical formulation and the analytical derivation of the full kinetic expressions of the pinch coefficients from the non-linear electrostatic gyrokinetic equation and discuss the linear limit and the physics implied. Section 3 presents and discusses the numerical results through parameter scans and compares this with observations documented in the literature. Section 4 summarizes the results.

\section{Gyrokinetic evaluation of particle transport contributions}

The particle continuity equation for an axisymmetric system, in stationary state and neglecting particle sources, reduces to the simple condition $\Gamma=0$, where $\Gamma$ is the particle flux of the considered species. In the following we neglect the neoclassical contribution and other sources to consider only the effect of turbulence, i.e. we have $\Gamma=\Gamma_{\text {turb }}=0$. Note however that the neoclassical pinch for electrons, notably the Ware pinch, can indeed be important in particular scenarios [29, 18]. Note that neglecting of the source term is relevant up to very large radius in experimental cases with only RF heating, for example in Ohmic TCV scenarios with ECH [30]. Moreover, the main effects of the Ware pinch and of a source can be added relatively easily in the framework presented here since they are essentially additive to the main driving terms sustaining a finite density gradient [31] .

In the following we shall see that $\Gamma_{\text {turb }}=0$ is generally found at $\partial \log n / \partial \rho \neq 0$, which means that the diagonal diffusive term in the flux expression is balanced by a convective term which is driven by the background turbulence and can provide a peaked or a hollow profile depending on the turbulence characteristics. The main goal of the theoretical development is to evaluate $\Gamma_{\text {turb }}$ and clarify the different mechanisms that drive the diffusive and the convective terms. The starting point is the non-linear gyrokinetic equation written in the ballooning representation as derived in Ref. [32], 
which we consider here for a generic species and neglecting magnetic perturbations. We first set the geometry to the simple concentric shifted-circles (or $s-\alpha$ ) magnetic equilibrium in the low aspect ratio limit. We write the gyrokinetic equation in matrix multiplication form:

$$
L \tilde{\mathrm{g}}+\boldsymbol{N}=\boldsymbol{W} \tilde{\Phi},
$$

where $\tilde{\mathbf{g}}, \tilde{\boldsymbol{\Phi}}$ are the vectors of fluctuations for the non-adiabatic part $\tilde{g}_{\mathbf{k}}$ of the distribution and of the electrostatic potential $\tilde{\Phi}_{\mathbf{k}}$ for each mode wavenumber $\mathbf{k}$ identified by the two independent components $\mathbf{k}=\left(k_{\mathrm{y}}, k_{\mathrm{x}}\right)$, with $x, y$ respectively the normal and the binormal to-the-field-line coordinates [33]. The matrix operators are defined as:

$$
\begin{aligned}
& \boldsymbol{L}_{\mathbf{k} \mathbf{k}^{\prime}}=\left[\frac{\partial}{\partial t}-\frac{v_{\|}}{R q} \frac{\partial}{\partial \theta}+i \omega_{\mathrm{d}}+\hat{C}\right]_{\mathbf{k}} \boldsymbol{I}_{\mathbf{k} \mathbf{k}^{\prime}}, \\
& \boldsymbol{N}=\sum_{\mathbf{k}^{\prime}+\mathbf{k}^{\prime \prime}=\mathbf{k}}\left[\left(k_{\mathrm{y}}^{\prime \prime} k_{\mathrm{x}}^{\prime}-k_{\mathrm{y}}^{\prime} k_{\mathrm{x}}^{\prime \prime}\right) J_{0 \mathbf{k}^{\prime \prime}} \tilde{\Phi}_{\mathbf{k}^{\prime \prime}} \tilde{g}_{\mathbf{k}^{\prime}}\right]=\mathbf{N}_{\mathbf{k k}^{\prime}} \tilde{g}_{\mathbf{k}^{\prime}}, \\
& \mathbf{N}_{\mathbf{k k}^{\prime}}\left(\mathbf{k}^{\prime \prime}=\mathbf{k}-\mathbf{k}^{\prime}\right)=\left(k_{\mathrm{y}}^{\prime \prime} k_{\mathrm{x}}^{\prime}-k_{\mathrm{y}}^{\prime} k_{\mathrm{x}}^{\prime \prime}\right) J_{0 \mathbf{k}^{\prime \prime}} \tilde{\Phi}_{\mathbf{k}^{\prime \prime}}, \\
& \boldsymbol{W}_{\mathbf{k k}^{\prime}}=\frac{Z e}{T}\left[\frac{\partial}{\partial t}+i \omega_{*}\right]_{\mathbf{k}} F_{0} J_{0 \mathbf{k}} \boldsymbol{I}_{\mathbf{k k}^{\prime}},
\end{aligned}
$$

where $\boldsymbol{I}$ is the diagonal identity matrix. $\theta$ is the parallel-to-the-field-lines coordinate. Equation (1) gives the evolution of the non-adiabatic part $\tilde{g}$ of the total gyro-averaged perturbed distribution function $\tilde{f}_{\mathbf{k}}=\tilde{g}_{\mathbf{k}}-Z e / T F_{0} J_{0 \mathbf{k}} \tilde{\Phi}_{\mathbf{k}}$. The usual definitions for the different quantities are:

$\omega_{\mathrm{d}}=-\left(v_{\|}^{2}+\frac{v_{\perp}^{2}}{2}\right) \frac{T k_{\mathrm{y}}}{v_{\mathrm{th}}^{2} Z e B_{0} R}\left[\cos \theta+\left(s \theta-\alpha \sin \theta+\frac{k_{\mathrm{x}}}{k_{\mathrm{y}}}\right) \sin \theta\right]$,

$\omega_{*}=\frac{T k_{\mathrm{y}}}{Z e B_{0} R}\left[\frac{\partial \log n}{\partial \rho}+\left(\frac{E}{T}-\frac{3}{2}\right) \frac{\partial \log T}{\partial \rho}\right]$

respectively for the magnetic curvature drift $\omega_{\mathrm{d}}$ and for the diamagnetic drift $\omega_{*}, F_{0}$ is the Maxwellian distribution, $J_{0 \mathbf{k}}$ is the zero-order Bessel function of argument $k_{\perp} \rho_{\mathrm{L}} v_{\perp} / v_{\text {th }}$ with $k_{\perp}=\sqrt{k_{\mathrm{y}}^{2}\left[1+(s \theta-\alpha \sin \theta)^{2}\right]+k_{\mathrm{x}}^{2}}$ the perpendicular wavenumber, $v_{\perp} / v_{\mathrm{th}}$ the perpendicular velocity normalized to the thermal velocity, and $\rho_{\mathrm{L}}$ the particle Larmor radius (for passing electrons $\rho_{\mathrm{L}}<<\rho_{\mathrm{i}}, \rho_{\mathrm{i}}$ being the ion Larmor radius, giving $J_{0 \mathbf{k}} \approx 1$ in the ITG-TEM wavelengths region, while for bounce-averaged trapped electrons the banana orbit is considered and $\rho_{\mathrm{L}} \sim \rho_{\mathrm{i}}$ ). $Z$ is the species charge normalized to the electron charge $e, T$ the species temperature, $R$ is the geometrical average major radius of the local flux surface, $q$ is the safety factor, $B_{0}$ the vacuum magnetic field at the plasma boundary geometrical axis, $E, v_{\|}, v_{\perp}$ respectively the particle energy, parallel and perpendicular velocity, $\rho$ is the normalized minor radius (or local aspect ratio) $\rho=r / R . \quad \boldsymbol{L}$ is the linear operator acting on the perturbed $\tilde{g}$, where collisionality is also contained in the form of a linear collisional operator $\hat{C}$, while $\boldsymbol{N}$ is the non-linear operator arising from the $\tilde{\mathbf{E}} \times \mathbf{B} \cdot \nabla \sim \mathbf{e}_{\|} \cdot \nabla \tilde{\Phi} \times \nabla$ advection of $\tilde{g}$, which causes mode coupling. The non-linear operator is represented as a matrix operation through the 
coupling matrix $\mathbf{N}$. Let us define a new matrix operator

$$
\mathbf{D}_{\mathbf{k k}^{\prime}}=\boldsymbol{L}_{\mathbf{k k}^{\prime}}+\mathbf{N}_{\mathbf{k k}^{\prime}} .
$$

We then rewrite the gyrokinetic equation (1) as a matrix system:

$$
\mathrm{D} \tilde{\mathbf{g}}=\tilde{\mathbf{P}},
$$

where $\tilde{\mathbf{P}}=W \tilde{\boldsymbol{\Phi}}$ is the vector with elements from the right-hand-side of equation (1). The solution of equation (5) is obtained through a formal matrix inversion operation:

$$
\tilde{\mathbf{g}}=\mathbf{D}^{-1} \tilde{\mathbf{P}} .
$$

Since $\omega_{*}$ is linear in the equilibrium logarithmic gradients $\partial \log n / \partial \rho$ and $\partial \log T / \partial \rho$, we can expand the vector $\tilde{\mathbf{P}}$ as:

$$
\tilde{\mathbf{P}}=\tilde{\mathbf{P}}_{\mathrm{n}} \frac{\partial \log n}{\partial \rho}+\tilde{\mathbf{P}}_{\mathrm{T}} \frac{\partial \log T}{\partial \rho}+\tilde{\mathbf{P}}_{\mathrm{P}}
$$

where

$$
\begin{aligned}
\tilde{\mathbf{P}}_{\mathrm{nk}} & =\left[\frac{k_{\mathrm{y}}}{B_{0} R} F_{0} J_{0 \mathbf{k}} \tilde{\Phi}_{\mathbf{k}}\right], \\
\tilde{\mathbf{P}}_{\mathrm{T} \mathbf{k}} & =\left[\frac{k_{\mathrm{y}}}{B_{0} R}\left(\frac{E}{T}-\frac{3}{2}\right) F_{0} J_{0 \mathbf{k}} \tilde{\Phi}_{\mathbf{k}}\right], \\
\tilde{\mathbf{P}}_{\mathrm{Pk}} & =\left[\frac{Z e}{T} F_{0} J_{0 \mathbf{k}} \frac{\partial \tilde{\Phi}_{\mathbf{k}}}{\partial t}\right],
\end{aligned}
$$

We now define $\tilde{\alpha}_{\mathrm{n}}=\mathbf{D}^{-1} \tilde{\mathbf{P}}_{\mathrm{n}}, \tilde{\alpha}_{\mathrm{T}}=\mathbf{D}^{-1} \tilde{\mathbf{P}}_{\mathrm{T}}$ and $\tilde{\alpha}_{\mathrm{P}}=\mathbf{D}^{-1} \tilde{\mathbf{P}}_{\mathrm{P}}$ to finally rewrite the solution (6) as:

$$
\tilde{\mathbf{g}}=\tilde{\alpha}_{\mathrm{n}} \frac{\partial \log n}{\partial \rho}+\tilde{\alpha}_{\mathrm{T}} \frac{\partial \log T}{\partial \rho}+\tilde{\alpha}_{\mathrm{P}} .
$$

Note that this decomposition is valid only in the local limit. If a profile variation other than the local gradient has to be taken into account, then a global model should be applied.

The turbulent flux $\Gamma_{\text {turb }}$ is produced by the cross-correlation between density and radial convection fluctuations:

$$
\Gamma_{\text {turb }}=<\tilde{n} \tilde{v}_{\mathbf{E} \times \mathbf{B}}^{\mathrm{r}}>_{\mathbf{k} \omega},
$$

where the superscript ' $r$ ' indicates the radial component of the advection velocity $\tilde{v}_{\mathbf{E} \times \mathbf{B}}=1 / B^{2} \tilde{\mathbf{E}} \times \mathbf{B}$. Replacing the solution from the gyrokinetic equation and the expression for the velocity we obtain:

$$
\Gamma_{\text {turb }}=A^{\prime} \frac{R}{L_{\mathrm{n}}}+B^{\prime} \frac{R}{L_{\mathrm{T}}}+C^{\prime},
$$

where we have defined the normalized inverse length scales for density $R / L_{\mathrm{n}}=$ $-\partial \log n / \partial \rho$ and for temperature $R / L_{\mathrm{T}}=-\partial \log T / \partial \rho$. The three coefficients $A^{\prime}, B^{\prime}, C^{\prime}$ 
are given by:

$$
\begin{aligned}
& A^{\prime}=\left\langle\Re \sum_{\mathbf{k}}\left[\frac{i k_{\mathrm{y}} \tilde{\Phi}_{\mathbf{k}}^{*}}{B_{0}}<J_{0 \mathbf{k}} \tilde{\alpha}_{\mathrm{nk}}>_{v}\right]\right\rangle, \\
& B^{\prime}=\left\langle\Re \sum_{\mathbf{k}}\left[\frac{i k_{\mathrm{y}} \tilde{\Phi}_{\mathbf{k}}^{*}}{B_{0}}<J_{0 \mathbf{k}} \tilde{\alpha}_{\mathrm{T} \mathbf{k}}>_{v}\right]\right\rangle, \\
& C^{\prime}=\left\langle\Re \sum_{\mathbf{k}}\left[\frac{i k_{\mathrm{y}} \tilde{\Phi}_{\mathbf{k}}^{*}}{B_{0}}<J_{0 \mathbf{k}} \tilde{\alpha}_{\mathrm{Pk}}>_{v}\right]\right\rangle,
\end{aligned}
$$

with $\langle\ldots\rangle_{v}$ being the proper velocity-space integration and the external brackets indicate flux-surface averaging. The stationary condition $\Gamma_{\text {turb }}=0$ provides a direct link between the normalized density gradient and the other two terms:

$$
\left[\frac{R}{L_{\mathrm{n}}}\right]_{\mathrm{stat}}=-C_{\mathrm{T}} \frac{R}{L_{\mathrm{T}}}-C_{\mathrm{P}},
$$

where $C_{\mathrm{T}}=B^{\prime} / A^{\prime}$ and $C_{\mathrm{P}}=C^{\prime} / A^{\prime}$ are the two pinch coefficients which relate the stationary normalized density gradient to the temperature profile through the thermodiffusive mechanism $\left(C_{\mathrm{T}} R / L_{\mathrm{T}}\right)$ and to another pinch mechanism identified in $C_{\mathrm{P}}$ which arises from the physics of the polarization current. The latter can also be interpreted as arising from the interplay between the parallel and the perpendicular dynamics when looking at the gyrokinetic equation for $\tilde{f}$, for which, in the RHS of equation (1): $\frac{\partial}{\partial t} \rightarrow \frac{v_{\|}}{R q} \frac{\partial}{\partial \theta}-i \omega_{\mathrm{d}}$.

It is worth noting that equation (13) is only apparently linear in the gradients: indeed both $C_{\mathrm{T}}$ and $C_{\mathrm{P}}$ are strongly influenced by $R / L_{\mathrm{n}}, R / L_{\mathrm{T}}$ since they enter as the drivers of the turbulent state. However, the equation becomes strictly linear for a species with negligible concentration (standardly called a 'tracer' or a passive species). The two pinch mechanisms $C_{\mathrm{T}}, C_{\mathrm{P}}$ appearing in equation (13) have been identified and discussed in previous works in both the non-linear and the linear regimes (see for instance Ref. [17] and references therein). However, a systematic study of how their interplay produces the self-consistent steady-state from equation (13) has not yet been addressed in details. We will address this issue in the next sections using the linear limit of the theory, which is discussed in the following subsection. We would like to point out that in Ref. [34] the authors discuss the non-linear interplay between the unstable and the stable branch of a given $\left(k_{\mathrm{y}}, k_{\mathrm{x}}\right)$ to provide a new genuinely non-linear inward pinch contribution in collisional TEM turbulence. This could be added in a quasi-linear model by assigning a mixing-length weight also to the stable modes, together with subdominant modes that are not seen by time evolutionary calculations but can be computed by a spectral code. These new contributions would appear as modifications in the three coefficients $A^{\prime}, B^{\prime}$ and $C^{\prime}$. In the same way sub-dominant modes, not seen by initial-value linear calculations, could be added to the model, after having being evaluated, for example, with an eigenvalue--solver [35]. 


\begin{tabular}{|c|c|c|}
\hline Sign & Mode type & Acronym \\
\hline$\omega_{\mathrm{R}}>0$ & Trapped Electron Mode & TEM \\
& Electron Temperature Gradient Mode & ETG \\
\hline$\omega_{\mathrm{R}}<0$ & Ion Temperature Gradient Mode & ITG \\
\hline
\end{tabular}

Table 1. Mode real frequency sign convention.

\subsection{Linear limit}

If we let the non-linear coupling matrix strength $\|\mathbf{N}\| \rightarrow 0$ we can evaluate the pinch coefficients directly as:

$$
\begin{aligned}
C_{\mathrm{T}}^{\mathrm{QL}} & =\frac{\sum_{\mathbf{k}}\left|\tilde{\Phi}_{\mathbf{k}}(0)\right|^{2} B_{\mathbf{k}}^{\prime}}{\sum_{\mathbf{k}}\left|\tilde{\Phi}_{\mathbf{k}}(0)\right|^{2} A_{\mathbf{k}}^{\prime}}, \\
C_{\mathrm{P}}^{\mathrm{QL}} & =\frac{\sum_{\mathbf{k}}\left|\tilde{\Phi}_{\mathbf{k}}(0)\right|^{2} C_{\mathbf{k}}^{\prime}}{\sum_{\mathbf{k}}\left|\tilde{\Phi}_{\mathbf{k}}(0)\right|^{2} A_{\mathbf{k}}^{\prime}},
\end{aligned}
$$

where 'QL' stands for quasi-linear, and

$$
\begin{aligned}
& A_{\mathbf{k}}^{\prime}=\left\langle\frac{1}{\left|\tilde{\Phi}_{\mathbf{k}}(0)\right|^{2}} \Re\left[\frac{k_{\mathbf{y}}^{2}}{B_{0}^{2} R}<J_{0 k} \boldsymbol{L}_{\mathbf{k k}}^{-1}\left(F_{0} J_{0 k} \tilde{\Phi}_{\mathbf{k}}\right)>_{v} \tilde{\Phi}_{\mathbf{k}}^{*}\right]\right\rangle, \\
& B_{\mathbf{k}}^{\prime}=\left\langle\frac{1}{\left|\tilde{\Phi}_{\mathbf{k}}(0)\right|^{2}} \Re\left[\frac{k_{\mathbf{y}}^{2}}{B_{0}^{2} R}<J_{0 k} \boldsymbol{L}_{\mathbf{k k}}^{-1}\left(F_{0}\left(\frac{E}{T}-\frac{3}{2}\right) J_{0 k} \tilde{\Phi}_{\mathbf{k}}\right)>_{v} \tilde{\Phi}_{\mathbf{k}}^{*}\right]\right\rangle, \\
& C_{\mathbf{k}}^{\prime}=\left\langle\frac{Z e}{T} \frac{1}{\left|\tilde{\Phi}_{\mathbf{k}}(0)\right|^{2}} \Re\left[\frac{k_{\mathbf{y}}}{B_{0}}<J_{0 k} \boldsymbol{L}_{\mathbf{k k}}^{-1}\left(F_{0} J_{0 k} \frac{\partial \tilde{\Phi}_{\mathbf{k}}}{\partial t}\right)>_{v} \tilde{\Phi}_{\mathbf{k}}^{*}\right]\right\rangle .
\end{aligned}
$$

The value of $\left|\tilde{\Phi}_{\mathbf{k}}(0)\right|^{2}$ is calculated as the value of $\left|\tilde{\Phi}_{\mathbf{k}}(\theta)\right|^{2}$ at $\theta=0$, which is usually near the maximum value for electrostatic modes with $k_{\mathrm{x}}=0$. With these definitions the three coefficients $A_{\mathbf{k}}^{\prime}, B_{\mathbf{k}}^{\prime}, C_{\mathbf{k}}^{\prime}$ do not depend on the absolute value of $\tilde{\Phi}$, avoiding the problem of the saturation regime. The relative dependence of $\tilde{\Phi}_{k}(\theta)$ on $\theta$, that depends on the shape of the eigenfunction, will affect the flux surface average. The $k$-dependence of the eigenfunction is also obtained from the linear solution. On the other hand the $k$-dependence of the absolute value, determined by $\left|\tilde{\Phi}_{\mathbf{k}}(0)\right|^{2}$, need to be given by a quasi-linear approximation. This is usually done by either the standard mixing length estimate, i.e. $\tilde{\Phi}_{k} \sim \gamma / k^{2}[36]$, or with a form such as to model nonlinear or experimental results $[37,20]$.

The linearized equation is solved in terms of simple plane waves $\sim e^{i(\mathbf{k x}-\omega t)}$ where $\omega=i \gamma+\omega_{\mathrm{R}}$ is the complex frequency, $\gamma$ is the growth rate and $\omega_{\mathrm{R}}$ the real angular rotation frequency. Since the magnetic curvature coupling term $\omega_{\mathrm{d}}$ and the driver $\omega_{*}$ for the electrons describe rotation in the positive direction, it follows that if $\omega_{R}>0$ then the mode is rotating in the electron diamagnetic direction, and if $\omega_{\mathrm{R}}<0$ the mode is rotating in the ion diamagnetic direction. We summarize this frequency convention in table 1 to show how to relate the sign of the real frequency to the type of instability. Using the plane waves decomposition we can obtain the quasi-linear expression of the two 
pinch coefficients which is summarized here for deeply trapped electrons $\left(v_{\|}=0\right)$, with a relative concentration $f_{\mathrm{t}}$, and for nearly-adiabatic passing electrons with a relative concentration $\left(1-f_{\mathrm{t}}\right)$ :

$$
\begin{gathered}
D_{0} C_{\mathrm{T}}=\sum_{\mathbf{k}} k_{\mathrm{y}}^{2}\left|\frac{e \tilde{\Phi}_{\mathbf{k}}}{T_{\mathrm{e}}}\right|^{2}\left[\sqrt{\frac{2}{\pi}} f_{\mathrm{t}} \int \overline{J_{0 k}^{2}} \frac{\hat{\gamma}\left(E-\frac{3}{2}\right) \sqrt{E} e^{-E}}{\left(\omega_{\mathrm{R}}-\bar{\omega}_{\mathrm{d}}\right)^{2}+\hat{\gamma}^{2}} d E\right. \\
\left.-\frac{\left(1-f_{\mathrm{t}}\right)}{2} \sqrt{\frac{\pi}{2}} \frac{1}{\left|k_{\| \mid} v_{\mathrm{th}}^{\mathrm{e}}\right|}\right], \\
D_{0} C_{\mathrm{P}}=-\frac{e B_{0} R}{T_{\mathrm{e}}} \sum_{\mathbf{k}} k_{\mathrm{y}}\left|\frac{e \tilde{\Phi}_{\mathbf{k}}}{T_{\mathrm{e}}}\right|^{2}\left[\sqrt{\frac{2}{\pi}} f_{\mathrm{t}} \int \overline{J_{0 k}^{2}} \frac{\left(\nu \omega_{\mathrm{R}}+\gamma \bar{\omega}_{\mathrm{d}}\right) \sqrt{E} e^{-E}}{\left(\omega_{\mathrm{R}}-\bar{\omega}_{\mathrm{d}}\right)^{2}+\hat{\gamma}^{2}} d E\right. \\
\left.+\left(1-f_{\mathrm{t}}\right) \sqrt{\frac{\pi}{2}} \frac{\omega_{\mathrm{R}}}{\left|k_{\|} v_{\mathrm{th}}^{\mathrm{e}}\right|}\right],
\end{gathered}
$$

where

$$
\begin{aligned}
& D_{0}=\sum_{\mathbf{k}} k_{\mathrm{y}}^{2}\left|\frac{e \tilde{\Phi}_{\mathbf{k}}}{T_{\mathrm{e}}}\right|^{2} {\left[\sqrt{\frac{2}{\pi}} f_{\mathrm{t}} \int \overline{J_{0 k}^{2}} \frac{\hat{\gamma} \sqrt{E} e^{-E}}{\left(\omega_{\mathrm{R}}-\bar{\omega}_{\mathrm{d}}\right)^{2}+\hat{\gamma}^{2}} d E\right.} \\
&\left.+\left(1-f_{\mathrm{t}}\right) \sqrt{\frac{\pi}{2}} \frac{1}{\left|k_{\|} v_{\mathrm{th}}^{\mathrm{e}}\right|}\right],
\end{aligned}
$$

and $\hat{\gamma}=\gamma+\nu$. The over-bar indicates that we have performed averaging over the mode structure and considered deeply trapped particles only, forcing $\mu B=E$. In this case $\overline{J_{0 k}^{2}}$, the Bessel function, is given by $\overline{J_{0 k}}=J_{0}\left(k_{\perp} \rho_{\mathrm{b}} \sqrt{2 E}\right), \rho_{\mathrm{b}}$ being the banana width, and the curvature drift by $\bar{\omega}_{\mathrm{d}}=f\left(s, k_{\mathrm{y}}, k_{\mathrm{x}}\right) \frac{T_{\mathrm{e}}}{e B_{0} R} E$. Note then that $\tilde{\Phi}_{\mathrm{k}}$ becomes a number. The particle energy $E$ is normalized to $T_{\mathrm{e}}$. Also each eigenfrequency $\gamma, \omega_{\mathrm{R}}$ varies with $\mathbf{k}=\left(k_{\mathrm{y}}, k_{\mathrm{x}}\right)$. Note that, since $\hat{\gamma}>0$, the diffusivity $D_{0}$ is explicitly shown to be positive definite as it should be, while $C_{\mathrm{T}}, C_{\mathrm{P}}$ can change sign.

\subsection{Basic physical mechanisms behind $C_{\mathrm{T}}$ and $C_{\mathrm{P}}$}

The two pinch mechanisms $C_{\mathrm{T}}$ and $C_{\mathrm{P}}$ can be understood in terms of basic particle kinetic physics and emerge as fundamental processes in plasma turbulence, directly looking at equations (16). We analyze the separate contributions in velocity space regions into the passing electrons contribution $(p)$ and the trapped electrons contribution $(t)$ (considered here as a whole as deeply trapped).

- Passing electrons pinch: due to their high parallel mobility, the passing electrons are essentially adiabatic (unless collisionality becomes strong enough or other effects create strong non-adiabaticity), except for those which have very low parallel velocity and for which $v_{\|} \approx \omega_{\mathrm{R}} / k_{\|}$, namely passing electrons diffuse due to parallel Landau damping. Because of the $E-3 / 2 T$ kernel in thermodiffusion, integrating with a delta function in $v_{\|}$and a Maxwellian in $v_{\perp}$ one finds that $C_{\mathrm{T}}^{(\mathrm{p})} \approx-1 / 2([26,38])$. For the other pinch with kernel $\sim \omega$, the contribution is $C_{\mathrm{P}}^{(\mathrm{p})} \approx-\omega_{\mathrm{R}} / k_{\mathrm{y}} e B_{0} R / T$, i.e. proportional to the mode real frequency, providing an outward directed pinch in ITG and an inward 
directed pinch in TEM-ETG, large in absolute value for $\left|\omega_{R}\right| \gg 1$. The physics of this pinch is particle convection due to compression in the parallel velocity (compare with the impurity pinch found in Ref. [39]).

- Trapped electrons pinch: trapped electrons average out the parallel dynamics due to magnetic mirror trapping, and thus their dynamics is dominated by the perpendicular drift $\omega_{\mathrm{d}}$. As for the passing electrons, for $C_{\mathrm{T}}^{(\mathrm{t})}$ the kernel is $\propto(E-3 / 2 T)$, i.e. low energy trapped electrons diffuse inwards, and high energy trapped electrons diffuse outwards. However in this case the resonance is not provided by Landau damping but by coupling between the mode real frequency and the drift, namely particles with $<\omega_{\mathrm{d}}>_{\theta} \approx \omega_{\mathrm{R}}$, where $<\ldots>_{\theta}$ is the average over the mode poloidal structure, which resonate with the wave and diffuse faster, providing a net convection. If $<\omega_{\mathrm{d}}>_{\theta}$ is positive, since $<\omega_{\mathrm{d}}>_{\theta} \propto E$, if $\omega_{\mathrm{R}}<0$ (ITG) low energy particles can resonate and we have a net inward convection. In the opposite case, if $\omega_{R}>0$ (TEM), the resonance can favor particles with higher energies, decreasing the net convection, and eventually leading to an inversion of the direction of the total pinch. Note also that for large negative values of $\omega_{\mathrm{R}}$ or for $\omega_{\mathrm{R}} \gg<\omega_{\mathrm{d}}>_{\theta}$ the resonance is weak and so the thermodiffusive pinch is expected to be reduced in both ITG or TEM turbulence. With regards to the other pinch $C_{\mathrm{P}}^{(\mathrm{t})}$, it is mainly driven by $<\omega_{\mathrm{d}}>_{\theta}$ and it is found to be inward directed for positive values of $\left\langle\omega_{\mathrm{d}}\right\rangle_{\theta}$. It is enhanced in TEM turbulence with respects to ITG turbulence due to the stronger resonance for trapped electrons. Finally, if the sign of $<\omega_{\mathrm{d}}>_{\theta}$ is reversed (for example for strongly reversed $q$ profiles or in stellarators) then the inward pinch becomes outward and viceversa.

- The effect of collisions on the trapped particles pinch: the real plasma has always a finite collisionality. Its effect on the pinch carried by trapped electrons (the passing electrons are assumed adiabatic in this calculation) can be extracted from equations (16). In particular an additional term appears in the $C_{\mathrm{P}}$ coefficient in the form:

$$
C_{\mathrm{P}}^{(\nu)} \approx-\frac{e B_{0} R}{T_{\mathrm{e}}} \frac{\omega_{\mathrm{R}}}{k_{\mathrm{y}}} \frac{\nu}{\gamma+\nu},
$$

where here $\nu$ is an 'averaged' collisionality (weighted on the Maxwellian distribution). It is interesting to observe that this pinch is outward directed for modes rotating in the ion direction, while it is inward directed for modes rotating in the electron direction. It saturates at large $\nu$, and its effect is reduced for increasing $T_{\mathrm{e}}$. In addition, since in general increasing $\nu$ has the effect of pushing $\omega_{\mathrm{R}}$ towards negative values (e.g. towards more ITG turbulence, due to its stabilizing effects on TEMs), the effect will be synergetic when $\omega_{\mathrm{R}}<0$, providing a strong enhancement of the outward pinch, while it will be counteracting when $\omega_{\mathrm{R}}>0$, producing little effect. This can explain why there is a strong collisionality effect in ITG-dominated regimes like $T_{\mathrm{e}} \approx T_{\mathrm{i}} \mathrm{H}$-modes as shown for example in Ref. [7], while no apparent $\nu$ scaling is observed in TEM-dominated regimes like $T_{\mathrm{e}} \gg T_{\mathrm{i}} \mathrm{L}$-modes, as discussed for TCV cases in Ref. [3]. Note also that in the presence of collisions the spectrum is somewhat larger than in the collisionless case, 
which requires taking into account a larger set of modes in $k_{\mathrm{y}}$ [40].

Now, the total $C_{\mathrm{T}}$ and $C_{\mathrm{P}}$ are provided by the two contributions, from trapped and passing particles, weighted by the respective diffusivity. For low collisionality $\left(\nu_{\mathrm{ei}} \ll \omega_{\mathrm{d}}\right)$ and moderate magnetic shear $(0.5 \lesssim s \lesssim 1.5)$, the passing electrons are nearly adiabatic and we expect a dominance of the trapped electron pinch. The situation can change with high collisionality or if adiabaticity is broken by other mechanisms (e.g. low magnetic shear, electromagnetic effects at high $\beta_{\mathrm{e}}$, collisions) like in the plasma edge, see Ref. [41] and references therein. We conclude this section by noticing that we did not mention the dynamics of the barely trapped electrons (trapped electrons with bouncing angles $\approx \pi$ ), since we assume that the electrons species can be divided in fully passing (no trapping), and deeply trapped $\left(v_{\|}=0\right)$ simply factorizing the fraction of each in front of the integrals. In general the contribution of the barely trapped electrons is important since they behave almost like the passing electrons (thermodiffusion appears to be not so sensitive on $\omega_{R}$ ) but their perpendicular diffusivity is comparable with that of the deeply trapped electrons. Comparing the numerical (full kinetic electrons) results with the simplified calculations presented in equations (16) we find that the essential physical

dependencies on parameters like $\nu$ or $\frac{T_{\mathrm{e}}}{T_{\mathrm{i}}}$ is retained in the latter case, but discrepancies in the absolute values are found. In this sense the simplified analytical approach is useful to understand the key physical mechanisms, but a full kinetic numerical treatment is required to obtain credible quantitative results, as done in the following sections.

\section{Numerical calculations of $C_{\mathrm{T}}$ and $C_{\mathrm{P}}$ and of the stationary state}

In this section we perform numerical calculation of turbulence linear spectra, phase shifts, and quasi-linear pinch coefficients $C_{\mathrm{T}}$ and $C_{\mathrm{P}}$ for different parameters scan to understand how a very general behavior of the stationary state of $R / L_{\mathrm{n}}$, provided by equation (13) (we drop the 'QL'), arises with respect to the background microinstabilities. As we consider only two bulk species, main ions and electrons, from plasma neutrality follows that we can evaluate the coefficients for electrons with the resulting stationary state consistent for both species. We thus evaluate $C_{\mathrm{T}}$ and $C_{\mathrm{P}}$ only for the electrons.

The numerical tool employed for the calculations is the initial value flux-tube gyrokinetic code GS2 [42, 43]. We use it in the linear, electrostatic version, with shifted-circles $s-\alpha$ equilibria. For the first parameter scan, a set of non-linear runs are performed to confirm the main results. Note that to discriminate the various linear contributions in the total flux, we adopt the test particle technique. We run the code with three species: main ions, bulk electrons, and the third species being a 'packet' of electrons with $n_{\mathrm{e}}^{\text {packet }}<<n_{\mathrm{e}}^{\text {bulk}}$, which with respect to quasi-neutrality satisfies $n_{\mathrm{e}}^{\text {packet }}+n_{\mathrm{e}}^{\text {bulk }}=n_{\mathrm{i}}$. For the electrons packet, we change the normalized gradients $\left(R / L_{\mathrm{n}}, R / L_{\mathrm{Te}}\right)$ in the combination $\left(R / L_{\mathrm{n}}, 0\right),\left(0, R / L_{\mathrm{Te}}\right)$, $(0,0)$, i.e. three simulations are launched for each case. Then, using the packet particle flux $\Gamma_{\mathrm{e}}^{\text {packet }}$ evaluated from the three simulations, we can find the three coefficients of the linear 
relation $\Gamma_{\mathrm{e}}^{\text {packet }}=A^{\prime} R / L_{\mathrm{n}}+B^{\prime} R / L_{\mathrm{Te}}+C^{\prime}$, where $A^{\prime}, B^{\prime}, C^{\prime}$ are in fact determined only by the bulk species for which no normalized gradient is changed (we stress that this is a linear relation exactly because it is a passive species). In this way the electrons packet does not change the background turbulence and we obtain the correct values of $A^{\prime}, B^{\prime}$ and $C^{\prime}$ at the parameters $\left(R / L_{\mathrm{n}}, R / L_{\mathrm{Te}}\right)_{\text {bulk }}$, since $A^{\prime}, B^{\prime}, C^{\prime}$ are independent on $\left(R / L_{\mathrm{n}}, R / L_{\mathrm{Te}}\right)_{\text {packet }}$. Changing the bulk electrons parameters would change the turbulence and leads to difficulties in deriving $A^{\prime}, B^{\prime}, C^{\prime}$.

In the next section we will show the numerical results in normalized form: the mode complex frequency $\omega=i \gamma+\omega_{\mathrm{R}}$ is scaled to $v_{\mathrm{th}}^{\mathrm{i}} / R$, i.e. $\hat{\omega}=i \hat{\gamma}+\hat{\omega}_{\mathrm{R}}=\omega R / v_{\mathrm{th}}^{\mathrm{i}}$ (we drop the hats in the following), where $v_{\mathrm{th}}^{\mathrm{i}}=\sqrt{T_{\mathrm{i}} / m_{\mathrm{i}}}$ is the ion thermal velocity. The 'ion' normalization is chosen to avoid spurious change in the mode frequency or in the wavenumber spectrum, when changing $T_{\mathrm{e}}$ at fixed $T_{\mathrm{i}}$, due solely to normalization. We also define the single $k$ particle flux $\Gamma_{\mathbf{k}}$ such that $\Gamma_{\text {turb }}=\sum_{\mathbf{k}} \Gamma_{\mathbf{k}}$, and the single $k$ phase-shift as $\tilde{\Gamma}_{\mathbf{k}}=\Gamma_{\mathbf{k}} /\left|\tilde{\Phi}_{\mathbf{k}}(0)\right|^{2}$. The quasi-linear single $k$ particle flux is then given by $\Gamma_{\mathbf{k}}^{\mathbf{q} \cdot l .}=\left|\tilde{\Phi}_{\mathbf{k}}(0)\right|^{2} \tilde{\Gamma}_{\mathbf{k}}$ where $\left|\tilde{\Phi}_{\mathbf{k}}(0)\right|^{2}$ is evaluated with equation (19).

\subsection{Choice of quasi-linear rule}

We define now the choice for the value of $\left|\tilde{\Phi}_{\mathbf{k}}(0)\right|^{2}$ to be used in equations (14) for the evaluation of the two pinch coefficients. Taking into account several results from previous works $[36,44,37,20,45]$ we choose to use the following rule:

$$
\left|\tilde{\Phi}_{\mathbf{k}}(0)\right|^{2}=A_{0}\left(\frac{\gamma}{<k_{\perp}^{2}>}\right)^{\zeta} \Delta k_{\mathrm{y}}
$$

where $\gamma_{\mathbf{k}}$ is the growth rate of the most unstable mode at wavelength $\mathbf{k}=\left(k_{\mathrm{y}}, 0\right)$ and $<k_{\perp}^{2}>$ is the perpendicular wavenumber averaged over the ballooning angle defined as in Ref. [37]:

$$
<k_{\perp}^{2}>=k_{\mathrm{y}}^{2} \frac{\int\left|\tilde{\Phi}_{\mathbf{k}}\right|^{2}\left[1+(s \theta-\alpha \sin \theta)^{2}\right] d \theta}{\int\left|\tilde{\Phi}_{\mathbf{k}}\right|^{2} d \theta} .
$$

The value of the constant $A_{0}$ does not matter in this context as the pinch coefficients are defined as ratios of absolute fluxes. The parameter $\Delta k_{\mathrm{y}}$ takes into account the choice of a non-uniform grid in $k_{\mathrm{y}}$. The numerical parameter $\zeta=2$ is used in the following and will be justified in the comparison with non-linear results. The choice of not considering a spectrum in $k_{\mathrm{x}}$ is not justified a priori since $k_{\mathrm{x}} \neq 0$ modes are fundamental in both non-linear mode coupling and turbulence saturation. However at this stage we prefer to leave the study of the full spectrum directly for non-linear simulations.

\subsection{Self-consistent stationary state}

Note that equation (13) has to be solved self-consistently for each parameters set with a scan in $R / L_{\mathrm{n}}$, as the coefficients themselves depend on $R / L_{\mathrm{n}}$. This means that, provided an input $R / L_{\mathrm{n}}^{\mathrm{In}}$ to the calculation of turbulence properties, the self-consistent stationary state is given when the $R / L_{\mathrm{n}}^{\text {Out }}=-C_{\mathrm{T}}\left(R / L_{\mathrm{n}}^{\mathrm{In}}\right) R / L_{\mathrm{Te}}-C_{\mathrm{P}}\left(R / L_{\mathrm{n}}^{\mathrm{In}}\right)=R / L_{\mathrm{n}}^{\mathrm{In}}=$ 


\begin{tabular}{|c|c|c|c|c|}
\hline Parameters & $R / L_{\mathrm{Te}}-R / L_{\mathrm{Ti}}$ & $\hat{\nu}-T_{\mathrm{e}} / T_{\mathrm{i}}$ & $s-T_{\mathrm{e}} / T_{\mathrm{i}}$ & $R / L_{\mathrm{Ti}}-\hat{\nu}$ \\
\hline$\epsilon$ & 0.12 & 0.12 & 0.12 & 0.12 \\
\hline$q$ & 1.4 & 1.4 & 1.4 & 2.8 \\
\hline $\mathrm{s}$ & 0.8 & 0.8 & - & -1 \\
\hline$R / L_{\mathrm{Te}}$ & - & 9 & 9 & 20 \\
\hline$R / L_{\mathrm{Ti}}$ & - & 9 & 7 & - \\
\hline$T_{\mathrm{e}} / T_{\mathrm{i}}$ & 1.5 & - & - & 2.8 \\
\hline$\hat{\nu}$ & 0 & - & 0.2 & - \\
\hline
\end{tabular}

Table 2. Parameters set.

$\left[R / L_{\mathrm{n}}\right]_{\text {stat }}$. At the same time we have the stationary value of $C_{\mathrm{T}}=C_{\mathrm{T}}\left(\left[R / L_{\mathrm{n}}\right]_{\text {stat }}\right)$ and similarly of $C_{\mathrm{P}}$. The stationary value $\left[R / L_{\mathrm{n}}\right]_{\text {stat }}$ is stable with respect to small perturbations if $\left[d X^{\text {out }} / d X^{\text {in }}\right]_{X=X_{\text {stat }}}>1$ where $X=R / L_{\mathrm{n}}$. In the following we will show the relevant quantities evaluated at the self-consistent state $\left[R / L_{\mathrm{n}}\right]_{\text {stat }}$. In addition, since a spectrum of modes is taken into account, thus ITG and TEM modes might be contributing to the total flux, we define a weighted averaged real frequency $\omega_{\mathrm{R}}^{\mathrm{QL}}$ as $\left(k=k_{\mathrm{y}} \rho_{\mathrm{i}}\right)$ :

$$
\omega_{\mathrm{R}}^{\mathrm{QL}}=\frac{\sum_{\mathbf{k}}\left|\tilde{\Phi}_{\mathbf{k}}(0)\right|^{2} \omega_{\mathrm{R} k}}{\sum_{\mathbf{k}}\left|\tilde{\Phi}_{\mathbf{k}}(0)\right|^{2}},
$$

We shall see that it helps to understand which type of turbulence is dominating. It can be interpreted as an average rotational frequency of the turbulent flow. If TEM dominates, then we expect $\omega_{\mathrm{R}}^{\mathrm{QL}} \gtrsim 0$. In the case of ITG-dominated turbulence, we expect $\omega_{\mathrm{R}}^{\mathrm{QL}} \lesssim 0$, even if the frequency itself has different signs at different values of $k_{\mathrm{y}} \rho_{\mathrm{i}}$. When we are in a mixed regime, where no single dominant instability can be identified, we find that $\left|\omega_{\mathrm{R}}^{\mathrm{QL}}\right| \approx 0$.

\subsection{Parameters set}

In this paper we consider transport related to the core plasma defined as the region as typically $0.3 \lesssim r / a \lesssim 0.8$. The parameters used in the following are typical of the mid-radius region $r / a \approx 0.5$ where the driving gradients and turbulent transport are usually relevant.

The following scans will be performed around fixed parameters as shown in table 2 . Note that the Shafranov-shift parameter $\alpha$ is calculated self-consistently for every case as $\alpha=-q^{2} d \beta / d \rho$ where $\beta=2 \mu_{0}\left(n_{\mathrm{e}} T_{\mathrm{e}}+n_{\mathrm{i}} T_{\mathrm{i}}\right) / B_{0}^{2}$ with $n_{\mathrm{e}}=n_{\mathrm{i}}=1\left[10^{19} \mathrm{~m}^{-3}\right]$, $T_{\mathrm{i}}=1[\mathrm{keV}]$ and $B_{0}=2[\mathrm{~T}]$. We also define a base case parameters set: $\epsilon=0.12$, $q=1.4, s=0.8, R / L_{\mathrm{Te}}=9, R / L_{\mathrm{Ti}}=9, T_{\mathrm{e}} / T_{\mathrm{i}}=1.5, \hat{\nu}=0, Z_{\text {eff }}=1$. The first four scans are devoted to study the relevant dependencies for typical monotonic $q$ scenarios, while the last scan can be applied to interpret electron Internal Transport Barrier scenarios with strongly reversed $q$-profile [16]. The normalized collisionality $\hat{\nu}$ is 
defined as $\hat{\nu}=\nu_{\mathrm{ei}} R / v_{\mathrm{th}}^{\mathrm{i}}$ where $\nu_{\mathrm{ei}}$ is the electron-ion collisional frequency from Coulomb scattering. With regards to the code numerical parameters that are kept fixed, we use 32 points for each $2 \pi$ turn in $\theta, 12$ poloidal periods, $\Delta t=0.03$ in units of $R / v_{\text {th }}^{\mathrm{i}}$, we set $k_{\mathrm{x}}=0$ and we solve for 13 values of $k_{\mathrm{y}} \rho_{\mathrm{i}}$ which cover the interval $0.08 \div 1.5$ in a logarithmic way, such that $\Delta k_{\mathrm{y}} \rho_{\mathrm{i}} \propto k_{\mathrm{y}} \rho_{\mathrm{i}}$.

\section{4. $R / L_{\mathrm{Te}}-R / L_{\mathrm{Ti}}$ scan, collisionless}

The first scan studies the main drivers of the ITG and of the temperature-driven TEM, causing a smooth transition between the two modes as the relative drivers strength $L_{\mathrm{Ti}} / L_{\mathrm{Te}}$ is changed. The relative weight between the thermodiffusive part of the total normalized density gradient, namely $-C_{\mathrm{T}} R / L_{\mathrm{Te}}$, and the other contribution $C_{\mathrm{P}}$, is also changed with $R / L_{\mathrm{Te}}$. The temperature normalized gradients are set at: $R / L_{\mathrm{Te}}=[5,7,9,11,13]$ and $R / L_{\mathrm{Ti}}=[6,9,12]$.

3.4.1. Linear simulations In figure 1 we show respectively: (a) the average real frequency $\omega_{\mathrm{R}}^{\mathrm{QL}}$, (b) the stationary value of $R / L_{\mathrm{n}}=\left[R / L_{\mathrm{n}}\right]_{\text {stat }}$, (c) the two pinch coefficients $C_{\mathrm{T}}$ and (d) $C_{\mathrm{P}}$, versus the parameter $L_{\mathrm{Ti}} / L_{\mathrm{Te}}$ for different values of $R / L_{\mathrm{Te}}$ (in the legend). Since $\omega_{\mathrm{R}}^{\mathrm{QL}}$ changes smoothly from negative to positive increasing $L_{\mathrm{Ti}} / L_{\mathrm{Te}}$, we conclude that the turbulent regime changes globally from ITG-dominated to TEMdominated increasing this parameter and figure $1(\mathrm{a})$ shows that $\omega_{\mathrm{R}}^{\mathrm{QL}}$ and $L_{\mathrm{Ti}} / L_{\mathrm{Te}}$ are good definitions to encapsulate this effect. Figure $1(\mathrm{~b})$ shows that $\left[R / L_{\mathrm{n}}\right]_{\text {stat }}$ has a maximum near $\omega_{\mathrm{R}}^{\mathrm{QL}} \approx 0$. Indeed, at fixed $R / L_{\mathrm{Te}},\left[R / L_{\mathrm{n}}\right]_{\text {stat }}$ increases when $\omega_{\mathrm{R}}^{\mathrm{QL}}$ approaches zero, either from the ITG domain $\left(\omega_{\mathrm{R}}^{\mathrm{QL}}<0\right)$ or from the TEM domain $\left(\omega_{\mathrm{R}}^{\mathrm{QL}}>0\right)$. A minimum in the particle flux $\Gamma$ at fixed $R / L_{\mathrm{n}}$ was also observed for $L_{\mathrm{Ti}} / L_{\mathrm{Te}} \approx 1$ in recent non-linear simulations [46]. Note also that the global increase in $\left[R / L_{\mathrm{n}}\right]_{\text {stat }}$ when changing $R / L_{\mathrm{Te}}$ at fixed $L_{\mathrm{Ti}} / L_{\mathrm{Te}}$ is due to the direct increase of the thermodiffusive pinch $-C_{\mathrm{T}} R / L_{\mathrm{Te}}$ at fixed $C_{\mathrm{T}}$. Indeed, $C_{\mathrm{T}}$ and $C_{\mathrm{P}}$ mainly depend on $L_{\mathrm{Ti}} / L_{\mathrm{Te}}$ as seen in figure $1(\mathrm{c}, \mathrm{d})$. As expected from the simple theoretical considerations done in subsection $(2.2), C_{\mathrm{T}}$ is inward directed and maximized in absolute value near $L_{\mathrm{Ti}} / L_{\mathrm{Te}} \approx 1$ (or rather $\omega_{\mathrm{R}}^{\mathrm{QL}} \approx 0$ ), and decreases strongly going to TEM-dominated turbulence at $L_{\mathrm{Ti}} / L_{\mathrm{Te}}>1$. It also means that trapped electrons dominate the pinch. On the other hand, $C_{\mathrm{P}}$ is found to be inward directed and growing higher in absolute value going into TEM-dominated turbulence, with values $\left|C_{\mathrm{P}}\right| \sim 1$. The interplay between the two pinches explains the behavior shown by $\left[R / L_{\mathrm{n}}\right]_{\text {stat }}$, and it is mainly due to the change in background turbulence, i.e. in $\omega_{\mathrm{R}}^{\mathrm{QL}}$, following the drivers ratio $L_{\mathrm{Ti}} / L_{\mathrm{Te}}$.

3.4.2. Detailed spectrum analysis It is interesting to check how the stationary state is made up by the interplay of different wavenumbers in the spectrum. We take as an example the stationary case with $R / L_{\mathrm{Te}}=R / L_{\mathrm{Ti}}=9$ of figure 1 (middle point of the diamonds curve), and show in figure 2: (a) the growth rate $\gamma$, (b) the phase velocity $\omega_{\mathrm{R}} /\left(k_{\mathrm{y}} \rho_{\mathrm{i}}\right),(\mathrm{c})$ the phase shift, and $(\mathrm{d})$ the quasi-linear flux per mode. The growth rate 
is maximum around $k_{\mathrm{y}} \rho_{\mathrm{i}} \approx 0.3$, while the value of $\gamma /<k_{\perp}^{2}>$ is maximum around $k_{\mathrm{y}} \rho_{\mathrm{i}} \approx 0.15$. Looking at the phase velocity $\omega_{\mathrm{R}} /\left(k_{\mathrm{y}} \rho_{\mathrm{i}}\right)$ we observe that the dominant modes are TEM at low wavenumbers and ITG at intermediate wavenumbers, while another electron mode branch appears in the short wavelength region. The phase-shift reveals that the transport is outward in the long wavelength TEM region, inward in the ITG region, and inward in the short wavelength TEM region. Finally, the quasi-linear flux per mode plot shows that the total zero flux is provided by a compensation of opposite contribution at different wavenumbers, namely outward flux in the low $k_{\mathrm{y}} \rho_{\mathrm{i}}$ region and inward flux in the high $k_{\mathrm{y}} \rho_{\mathrm{i}}$ region. This compensation evolves when the plasma parameters are modified. This is why a summation over the $k_{\mathrm{y}} \rho_{\mathrm{i}}$ spectrum is needed and why the definition of $\omega_{\mathrm{R}}^{\mathrm{QL}}$, equation (21), is very useful to be able to follow the main background turbulence.

\subsubsection{Comparison with non-linear results We now compare the quasi-linear results} with non-linear simulations performed with the GYRO code [47]. The resolution used in these simulations is of 32 toroidal modes, a box size of $100 \rho_{\mathrm{s}}$ in $x$ and $135 \rho_{\mathrm{s}}$ in $y$, with $\Delta x / \rho_{\mathrm{s}}=0.5$, and 128 grid points in velocity space. We employ the base case with $R / L_{\mathrm{Te}}=9$ and perform a scan in $R / L_{\mathrm{Ti}}=[4,9,14]$ at two values of $R / L_{\mathrm{n}}=[3,5]$. In figure 3 we show the results for the non-linear particle flux (plotted in arbitrary units, solid lines) as a function of $R / L_{\mathrm{Ti}}$ for the two $R / L_{\mathrm{n}}$ values (legend). The quasi-linear flux (dashed lines) is also shown for comparison. Let us first look at the result for $R / L_{\mathrm{Ti}}=9$ and $R / L_{\mathrm{Ti}}=14\left(R / L_{\mathrm{Ti}} \geq R / L_{\mathrm{Te}}\right)$. The non-linear $\Gamma$ is slightly inward for $R / L_{\mathrm{n}}=3$ and outward for $R / L_{\mathrm{n}}=5$. Therefore the predicted quasi-linear $\left[R / L_{\mathrm{n}}\right]_{\text {stat }}$ is between 3 and 5 , probably closer to 3 . The quasi-linear result predicts $\left[R / L_{\mathrm{n}}\right]_{\text {stat }} \approx 4$ for these cases. They correspond to the two left points in figure 1(b) for the diamonds $\left(R / L_{\mathrm{Te}}=9\right)$. As seen from figure $1(\mathrm{~b})$, the predicted $\left[R / L_{\mathrm{n}}\right]_{\text {stat }}$ is much lower when $L_{\mathrm{Ti}} / L_{\mathrm{Te}}>1$. We see that the non-linear result is consistent with this quasi-linear result since the non-linear $\Gamma$ is positive at $R / L_{\mathrm{Ti}}=4$ even for $R / L_{\mathrm{n}}=3$. We also see that the quasi-linear result in figure 2 follows well the non-linear results. We have used $\zeta=2$ in equation (19) which gives the best overall agreement and we keep this value in all the scans presented in this paper.

\section{5. $\hat{\nu}-T_{\mathrm{e}} / T_{\mathrm{i}} \operatorname{scan}$}

The previous scans have been done in a collisionless regime $(\hat{\nu}=0)$. However the plasma has always a finite collisionality whatever small it is. From the theoretical point of view it has been found to have a strong effect on both the instabilities properties and on the turbulent pinch itself [9]. In some experimental regimes $\hat{\nu}$ appears to be the only relevant parameter in regression studies from databases of $\mathrm{H}$-mode plasmas from different machines $[7,48,49]$. To understand this behavior we perform a set of simulations with: $\hat{\nu}=[0,0.02,0.1,0.3,0.8]$ and $T_{\mathrm{e}} / T_{\mathrm{i}}=[1.1,1.5,2.5]$. In figure 4 we show the same quantities as in figure 1 , now for different values of $T_{\mathrm{e}} / T_{\mathrm{i}}$ (legend) and $\hat{\nu}(\mathrm{x}-$ 
axis). The stationary, averaged values, shown in figure 4, show an interesting behavior: the average frequency $\omega_{\mathrm{R}}^{\mathrm{QL}}$ in (a) goes deeply into the ITG regime as $\hat{\nu}$ increases, and it shifts up to TEM as $T_{\mathrm{e}} / T_{\mathrm{i}}$ increases. This comes from the fact that TEM are stabilized with increasing collisionality and destabilized with increasing $T_{\mathrm{e}} / T_{\mathrm{i}}[8,14]$. The value of $\left[R / L_{\mathrm{n}}\right]_{\text {stat }}$ shown in (b) is strongly decreased by $\hat{\nu}$ (touching negative values) for $T_{\mathrm{e}} / T_{\mathrm{i}} \approx 1$, while higher values of the temperature ratio weaken this effect. At $T_{\mathrm{e}} / T_{\mathrm{i}}=2.5$ there is no significant effect from $\hat{\nu}$. This behavior can be understood looking at $C_{\mathrm{T}}$ and $C_{\mathrm{P}}$ in figure $4(\mathrm{c}, \mathrm{d})$. The thermodiffusion coefficient is essentially constant (in particular for $\left.T_{\mathrm{e}} / T_{\mathrm{i}}=1.5\right)$, while $C_{\mathrm{P}}$ is strongly influenced by $\hat{\nu}$ : increasing $\hat{\nu}, C_{\mathrm{P}}$ becomes less negative and even outward directed and large for $T_{\mathrm{e}} / T_{\mathrm{i}} \approx 1$. For $T_{\mathrm{e}} / T_{\mathrm{i}}=2.5$ this decrease is less evident and is well compensated by the slight change in $C_{\mathrm{T}}$. For lower values of $T_{\mathrm{e}} / T_{\mathrm{i}}$, the change in $C_{\mathrm{P}}$ dominates and we observe a reduction in $\left[R / L_{\mathrm{n}}\right]_{\text {stat }}$, larger at $T_{\mathrm{e}} / T_{\mathrm{i}} \approx 1$. Again this behavior is related to the collisions-induced pinch term, equation (18), and confirms the interpretation given at the end of paragraph (2.2). In particular note that the effect of collisions on $C_{\mathrm{P}}$ can be guessed by comparing the strength of the two terms $\left|\omega_{\mathrm{R}} \hat{\nu}\right| \div\left|\gamma \omega_{\mathrm{d}}\right|$. In case of turbulence being near the transition, we find $\left|\omega_{\mathrm{R}}\right| \approx 0$ and thus, even at high $\hat{\nu}$, the curvature drift term dominates, providing an inward directed convection given by $C_{\mathrm{P}}$ (e.g. diamond curves in figure $4(\mathrm{~d})$ ). On the other hand, for strong ITG turbulence (e.g. circle curves in figure 4(d)), the frequency is large and negative, thus one finds $\left|\omega_{\mathrm{R}} \hat{\nu}\right| \gtrsim\left|\gamma \omega_{\mathrm{d}}\right|$, which gives a dominant positive (outward) contribution to $C_{\mathrm{P}}$.

\section{6. $s-T_{\mathrm{e}} / T_{\mathrm{i}}$ scan, collisional}

Magnetic shear is another parameter which experimental dependence is debated. We now show that the predicted dependence can drastically change when the turbulent regime is changed. To this purpose we perform a set of simulations with: $R / L_{\mathrm{Te}}=9$, $R / L_{\mathrm{Ti}}=7, \hat{\nu}=0.2$ and $s=[0.5,0.8,1.1,1.4,1.7]$ and $T_{\mathrm{e}} / T_{\mathrm{i}}=[1.1,1.5,2.5]$. The results are shown in figure 5 as done for the previous scans. As can be seen from the different plots the effect of shear and of the temperature ratio is highly non-trivial. In figure 5(a) we observe again the effect of pushing the frequency up with $T_{\mathrm{e}} / T_{\mathrm{i}}$ towards TEM regime. In fact, comparing the behavior of $\omega_{\mathrm{R}}^{\mathrm{QL}}$ and of $\left[R / L_{\mathrm{n}}\right]_{\text {stat }}$, figure $4(\mathrm{a}, \mathrm{b})$, we see that at low $T_{\mathrm{e}} / T_{\mathrm{i}}$ we are in ITG-dominated regime, and a variation of magnetic shear results in a variation of $\omega_{\mathrm{R}}^{\mathrm{QL}}$, and an almost proportional variation of $\left[R / L_{\mathrm{n}}\right]_{\text {stat }}$. In particular increasing $s$, we move downward in frequency and thus we decrease $\left[R / L_{\mathrm{n}}\right]_{\text {stat }}$. On the other hand, at high value of $T_{\mathrm{e}} / T_{\mathrm{i}}$, we are in the mixed regime (where $\omega_{\mathrm{R}}^{\mathrm{QL}} \approx 0$ ), and no clear variation of $\omega_{\mathrm{R}}^{\mathrm{QL}}$ with $s$ is observed. Instead $\left[R / L_{\mathrm{n}}\right]_{\text {stat }}$ increases monotonically with magnetic shear. To understand from which mechanism this behavior arises, we look at the two pinch coefficients $C_{\mathrm{T}}$ and $C_{\mathrm{P}}$, figure $5(\mathrm{c}, \mathrm{d})$. While $C_{\mathrm{T}}$ is essentially increasing in absolute value with magnetic shear, there is a striking difference in the behavior of $C_{\mathrm{P}}$ with magnetic shear depending on the value of $T_{\mathrm{e}} / T_{\mathrm{i}}$ : at low values of this parameter, $C_{\mathrm{P}}$ is outward directed and becomes larger increasing $s$, while at high 
values of the temperature ratio it becomes inward directed and small in absolute value. We thus conclude that a positive proportionality between the local magnetic shear and the stationary $R / L_{\mathrm{n}}$ exists only when the turbulence regime is not dominantly ITG but is in a mixed situation for which $C_{\mathrm{P}}$ stays inward directed (or small in absolute value). Note that in this case this shear dependence comes from the effect of $s$ on $C_{\mathrm{T}}$ and not from $C_{\mathrm{P}}$. In collisional plasmas this regime is found at higher values of $T_{\mathrm{e}} / T_{\mathrm{i}}$ with respect to a collisionless situation. On the other hand, in ITG-dominated turbulence $\left[R / L_{\mathrm{n}}\right]_{\text {stat }}$ does not depend much on $s$ or decreases with increasing $s$ due to a strong outward contribution from $C_{\mathrm{P}}$.

\section{7. $R / L_{\mathrm{Ti}}-\hat{\nu}$ scan in eITB scenario}

The parameters set for this scan are taken from a typical plasma obtained in the electron Internal Transport Barrier scenario (eITB) in the TCV tokamak [16, 50]. The main characteristics are the large local normalized gradients in electron temperature and density $\left(R / L_{\mathrm{n}} \sim 9 \sim 0.45 R / L_{\mathrm{Te}}\right)$ and the strong reversed $q$ profile that can provide $q \sim 3$ and $s \lesssim-1$ in the barrier region. However the ion temperature is not known with precision and a scan in $R / L_{\mathrm{Ti}}$ is useful to look at the predictions of the theoretical model with respect to this parameter. Collisionality in the eITB is usually of order $\hat{\nu} \sim 10^{-2}$ and it can have an effect on the TEM instability, and thus will be scanned. These two parameters are taken as: $R / L_{\mathrm{Ti}}=[0.3,0.45,0.6,0.75,0.9] R / L_{\mathrm{Te}}$ and $\hat{\nu}=[0,0.02,0.04]$. In the same fashion as previous subsections, we plot the relevant results in figure 6 . Looking at $\omega_{\mathrm{R}}^{\mathrm{QL}}$, figure $6(\mathrm{a})$, we see that there is a smooth transition from TEM to ITG turbulence increasing $R / L_{\mathrm{Ti}}$ at fixed $\hat{\nu}$. The point where $\omega_{\mathrm{R}}^{\mathrm{QL}} \approx 0$ shifts to lower values of $R / L_{\mathrm{Ti}}$ increasing $\hat{\nu}$. This automatically shifts (for the physical reason already discussed previously) the maximal value of $\left[R / L_{\mathrm{n}}\right]_{\text {stat }}$, as a function of $R / L_{\mathrm{Ti}}$ down in $R / L_{\mathrm{Ti}}$ for increasing $\hat{\nu}$, figure $5(\mathrm{~b})$. Note that, as $R / L_{\mathrm{Te}}=20$, the $\left[R / L_{\mathrm{n}}\right]_{\mathrm{stat}}$ is essentially given by $\left[R / L_{\mathrm{n}}\right]_{\text {stat }} \approx-C_{\mathrm{T}} R / L_{\mathrm{Te}}$, while $C_{\mathrm{P}}$ is practically negligible, as seen in figure $5(\mathrm{~d})$, all along the scan. So, for these parameters, the stationary state is driven almost exclusively by thermodiffusion, and thus strongly linked to the dominant instability. It is maximized when $\omega_{\mathrm{R}}^{\mathrm{QL}} \approx 0$, at a values of $\left|C_{\mathrm{T}}\right| \approx 0.3$, figure $5(\mathrm{c})$.

\subsection{Addressing the experimental observations}

Now let us discuss the relevance of these results with respect to some issues in interpreting experimental results. In figure $7(\mathrm{a})$ we compile the stationary normalized density gradients versus $-\omega_{\mathrm{R}}^{\mathrm{QL}}$, such that the transition from a TEM to an ITG dominated regime is read from left to right, for the $\hat{\nu}-\frac{T_{\mathrm{e}}}{T_{\mathrm{i}}}$ scan (solid symbols: circles, right triangles, diamonds) and the $s-\frac{T_{\mathrm{e}}}{T_{\mathrm{i}}}$ scan (open symbols: pentagrams, hexagrams, squares, crosses). We also add points from two additional $s-\hat{\nu}$ scans performed at $R / L_{\mathrm{Te}}=[10,12], R / L_{\mathrm{Ti}}=[6,7]$ to put in evidence the dependencies in the TEM branch (smaller open symbols: left triangles, up triangles, stars).

The significant decrease of $\left[R / L_{\mathrm{n}}\right]_{\text {stat }}$ with $\left(-\omega_{\mathrm{R}}^{\mathrm{QL}}\right)$ in the ITG-dominated regime is 
consistent with the well-known decrease of density peaking with collisionality observed in several devices in the $\mathrm{H}$-mode phases [10] (figure 1 of Ref. [7]). In figure 7(a), the arrow indicates that $\hat{\nu}$ is increased going towards positive $\left(-\omega_{\mathrm{R}}^{\mathrm{QL}}\right)$ values.

On the other hand, L-modes show an increase of the density peaking with shear $\left(l_{i}\right)$, as seen in figure 7 of Ref. [7], irrespective of the collisionality. However these discharges are near the ITG-TEM transition [7], due to the higher $T_{\mathrm{e}} / T_{\mathrm{i}}$ values. They correspond therefore to the data indicated with vertical arrows labelled 's' in figure 7 (a) which have $\left[R / L_{\mathrm{n}}\right]_{\text {stat }}$ increasing with $s$ near $-\omega_{\mathrm{R}}^{\mathrm{QL}} \lesssim 0$.

The L-mode behavior in AUG has been shown to have cases with either increasing or decreasing density peaking with collisionality [9]. The measured $R / L_{\mathrm{n}}$ versus $\nu_{\text {eff }}$ (figure 3a of Ref. [9]) shows a striking resemblance with our predicted stationary $R / L_{\mathrm{n}}$ versus $\left(-\omega_{\mathrm{R}}^{\mathrm{QL}}\right)$ shown in figure $7(\mathrm{a})$. Since $\left(-\omega_{\mathrm{R}}^{\mathrm{QL}}\right)$ increases with collisionality, we see that our quasi-linear predictions can explain many apparently contradicting observed behaviors when both ITG and TEM regimes are considered together.

In the TEM-dominated regime, left part of figure $7(\mathrm{a})$, the arrow ' $\hat{\nu}$ ' indicates that one can have situations of increasing $\left[R / L_{\mathrm{n}}\right]_{\text {stat }}$ with increasing collisionality. This can happen for example if the starting point is clearly in the TEM regime. Increasing collisionality will lead to a reduction of the TEM and turbulence will move towards $\omega_{\mathrm{R}}^{\mathrm{QL}} \approx 0$, therefore increasing $\left[R / L_{\mathrm{n}}\right]_{\text {stat }}$. This could explain the recent results shown in Ref. [13]. Note that increasing $T_{\mathrm{e}} / T_{\mathrm{i}}$ would strengthen the TEM and therefore counteract this effect.

In order to be able to draw more general predictions we show the values of $C_{\mathrm{T}}$ and $C_{\mathrm{P}}$ in figure $7(\mathrm{~b}, \mathrm{c})$ for the cases shown in figure $7(\mathrm{a})$. The first point is that the main dependence of both terms is on $\omega_{\mathrm{R}}^{\mathrm{QL}}$. This is particularly true for $C_{\mathrm{P}}$ which does not depend much on other parameters, like $s$, at fixed $\omega_{\mathrm{R}}^{\mathrm{QL}}$, figure $7(\mathrm{c})$. Moreover, $C_{\mathrm{P}}$ is small and not very important in TEM regime and near $\omega_{\mathrm{R}}^{\mathrm{QL}} \approx 0$. It becomes significant and outward directed in clear ITG regime, mainly due to the collisionality effect as explained in paragraph 2.2. The term $C_{\mathrm{T}}$ is essentially always inward and $\left|C_{\mathrm{T}}\right|$ is maximum near the ITG-TEM transition and much smaller in the TEM regime. Since $R / L_{\mathrm{Te}}$ is easily of order 10 , in particular in ECH plasmas [51], $-C_{\mathrm{T}} R / L_{\mathrm{Te}}$ is of order 4 for $\left(-C_{\mathrm{T}}\right) \approx 0.4$. Nevertheless, $C_{\mathrm{T}}$ also depends on shear and collisionality, at the same $\omega_{\mathrm{R}}^{\mathrm{QL}}$, which explains the wider vertical spread of data in figure $7(\mathrm{~b})$ as compared with figure $7(\mathrm{c}) \cdot \omega_{\mathrm{R}}^{\mathrm{QL}}$ is calculated using equation (21) and $\zeta=2$ in equation (19). We have checked that the results shown in figure 7 are similar using a rule as proposed in Ref. [20].

A final remark on this section is that an ultimate test for the model would be to compare experimentally measured turbulent frequencies at low and medium $k_{\mathrm{y}} \rho_{\mathrm{i}}$ values, with the prediction from the codes to check if the experimental dependencies are really due to the underlying turbulent regime [52]. 


\section{Conclusions}

In this work, theoretical results about fundamental mechanisms of turbulent particle convection for Tokamak plasmas have been presented, together with simulation results to understand the main dependencies and general behavior of the stationary normalized density gradient with respect to key plasma parameters. The numerical calculations employ a quasi-linear model to evaluate the pinch coefficients $C_{\mathrm{T}}$, for thermodiffusion, and $C_{\mathrm{P}}$, the other pinch term, from the linear spectrum. A quasi-linear averaged mode frequency $\omega_{\mathrm{R}}^{\mathrm{QL}}$ is also evaluated to identify the turbulent regime, either ITG or TEM (or mixed in the transition regime when $\omega_{\mathrm{R}}^{\mathrm{QL}} \approx 0$ ).

We find that $C_{\mathrm{T}}$ is inward directed and maximal in absolute value at $\omega_{\mathrm{R}}^{\mathrm{QL}} \approx 0$, decreasing slightly in ITG and strongly in TEM for the trapped electrons contribution, while the passing electron pinch is almost constant (and directed inwards) and small, albeit increasing with collisionality in weight. $C_{\mathrm{P}}$ is found to become more negative increasing $\omega_{\mathrm{R}}^{\mathrm{QL}}$, while it becomes large and outward directed for collisional plasmas in ITG. The interplay of the two explains why the stationary state is found to be maximal at $\omega_{\mathrm{R}}^{\mathrm{QL}} \approx 0$ whatever parameter scan. In addition an almost linear proportionality on magnetic shear of $\left[R / L_{\mathrm{n}}\right]_{\text {stat }}$ is found at fixed $\omega_{\mathrm{R}}^{\mathrm{QL}}$, due to a change in $\omega_{\mathrm{d}}^{\mathrm{QL}}$, only when turbulence is in a mixed ITG-TEM regime with no dominance of the ITG branch. A few non-linear runs with GYRO show a qualitative agreement with the behavior found for the collisionless normalized temperature gradients scan.

The comparison of all our scans and of our results with experimental observations confirms that the dominant effect that determines, through turbulent effects, $\left[R / L_{\mathrm{n}}\right]_{\text {stat }}$, is if the turbulence regime is near the ITG-TEM transition or not. Indeed, this is usually sufficient to predict the evolution of the local density peaking with plasma parameters. This is why the parameter $\omega_{\mathrm{R}}^{\mathrm{QL}}$, equation (21), is particularly useful when analysing a particular case. Since the predicted $R / L_{\mathrm{n}}$ is maximal near $\omega_{\mathrm{R}}^{\mathrm{QL}} \approx 0$ one can expect an increasing density peaking when moving towards $\omega_{\mathrm{R}}^{\mathrm{QL}}=0$ and decreasing otherwise. For example in ECH eITBs [16] a significant thermodiffusive pinch is observed when stabilizing TEM and this moving from $\omega_{\mathrm{R}}^{\mathrm{QL}} \gg 0$ towards $\omega_{\mathrm{R}}^{\mathrm{QL}}=0$. In typical $\mathrm{H}-$ modes, increasing collisionality tends to lead towards more ITG-dominated turbulence, $\omega_{\mathrm{R}}^{\mathrm{QL}}$ moves away from zero and $R / L_{\mathrm{n}}$ decreases. Moreover, increasing $T_{\mathrm{e}} / T_{\mathrm{i}}$ increases TEM and thus $\omega_{\mathrm{R}}^{\mathrm{QL}}$. Starting from $\omega_{\mathrm{R}}^{\mathrm{QL}}<0$ (ITG) then $R / L_{\mathrm{n}}$ can increase because $\omega_{\mathrm{R}}^{\mathrm{QL}}$ moves towards zero. Instead, starting from a mixed or TEM regime, like with ECH, then one moves towards $\omega_{\mathrm{R}}^{\mathrm{QL}} \gg 0$ (strong TEM) and $R / L_{\mathrm{n}}$ decreases significantly.

Our results also explain why one can have a strong shear dependence of the local density peaking in some cases and not in others. This is due to both the dependence of $C_{\mathrm{T}}$ on $s$ when $\omega_{\mathrm{R}}^{\mathrm{QL}} \approx 0$, which can lead to increasing $\left[R / L_{\mathrm{n}}\right]_{\text {stat }}$ with increasing $s$, and to the dependence of $\omega_{\mathrm{R}}^{\mathrm{QL}}$ on shear in ITG-dominated turbulence which tends to decrease $\left[R / L_{\mathrm{n}}\right]_{\text {stat }}$, figure 5 and figure $7(\mathrm{a})$.

This relatively 'simple' way to encapsulate very different situations through the dependence of $C_{\mathrm{T}}$ and $C_{\mathrm{P}}$ on $\omega_{\mathrm{R}}^{\mathrm{QL}}$ is very appealing. It needs to be compared 
in more details with specific experimental data and with situations where ETG are also important. More quantitative predictions of course require non-linear simulations. However these results should allow one to better, or easier, understand both experimental observations and non-linear simulations.

Finally it should be stressed that the present simulations and analysis pertain only to core particle transport but might help in understanding edge turbulent transport. Moreover, we have considered the predicted source-free turbulence-driven stationary logarithmic density gradient. However the effect of the neoclassical Ware pinch and of core particle sources are essentially additive with respect to $\left[R / L_{\mathrm{n}}\right]_{\text {stat }}[31]$ and thus our predictions should be useful in these cases as well.

\section{Acknowledgements}

The authors thank M. Kotschenreuther and W. Dorland for having made available the GS2 code, together with J. Candy and R. E. Waltz for providing the GYRO code. The GS2 simulations have been run on the PLEIADES2 cluster at EPFL, Lausanne. This work has been supported in part by the Swiss National Science Foundation.

[1] G. T. Hoang et al., Phys. Rev. Lett. 90, 155002 (2003)

[2] I. Furno, H. Weisen and TCV team, Phys. Plasmas 45, 735 (2003)

[3] A. Zabolotsky and H. Weisen, Plasma Phys. Control. Fusion 45, 735 (2003)

[4] G. T. Hoang et al., Nucl. Fusion 46, 306 (2006)

[5] D. R. Baker et al., Nucl. Fusion 40, 1003 (2000)

[6] H. Weisen et al., Nucl. Fusion 42, 136 (2002)

[7] H. Weisen et al., Nucl. Fusion 45, L1 (2005)

[8] C. Angioni et al., Phys. Rev. Lett. 90, 205003 (2003)

[9] C. Angioni et al., Phys. Plasmas 12, 112310 (2005)

[10] M. Greenwald et al., Nucl. Fusion 47, L26 (2007)

[11] M. Romanelli et al., Plasma Phys. Control. Fusion 49, 935 (2007)

[12] H. Takenaga et al., Nucl. Fusion 48, 075004 (2008)

[13] K. A. Razumova et al., Plasma Phys. Control. Fusion 50, 105004 (2008)

[14] C. Angioni et al., Nucl. Fusion 44, 827 (2004)

[15] A. Zabolotsky and H. Weisen, Plasma Phys. Control. Fusion 46 5, 594 (2006)

[16] E. Fable et al., Plasma Phys. Control. Fusion 48, 1 (2006)

[17] X. Garbet et al., Phys. Rev. Lett. 91, 035001 (2005)

[18] D. R. Ernst et al., Phys. Plasmas 11 5, 2637 (2004)

[19] C. Estrada-Mila, J. Candy and R. E. Waltz, Phys. Plasmas 13, 074505 (2006)

[20] C. Bourdelle et al., Phys. Plasmas 14 (11), 112501 (2007)

[21] B. Coppi and C. Spight, Phys. Rev. Lett. 41, 551 (1978)

[22] P. W. Terry, Phys. Fluids B 1, 1932 (1989)

[23] R. E. Waltz and R. R. Dominguez, Phys. Fluids B 1, 1935 (1989)

[24] J. Nycander and V. V. Yankov, Phys. Plasmas 8 2, 2874 (1995)

[25] D. R. Baker and M. N. Rosenbluth, Phys. Plasmas 5, 2936 (1998)

[26] F. Miskane et al., Phys. Plasmas 10, 4197 (2000)

[27] D. R. Baker, Phys. Plasmas 11, 992 (2004)

[28] X. Garbet et al., Phys. Plasmas 12, 082511 (2005)

[29] J. Stober et al., Nucl. Fusion 43, 1265 (2003)

[30] A. Zabolotsky et al., Nucl. Fusion 46, 594 (2006) 
[31] E. Fable, PhD These, These $\mathrm{N}^{o} 4334$, EPFL, Lausanne (2009)

[32] E. A. Frieman and L. Chen, Phys. Fluids 25, 502 (1982)

[33] M. A. Beer, S. C. Cowley and G. W. Hammett, Phys. Plasmas 2, 2687 (1995)

[34] P. W. Terry and R. Gatto, Phys. Plasmas 13, 062309 (2006)

[35] F. Merz and F. Jenko, The 4th IAEA-TM on the Theory of Plasma Instabilities, I-21 (2009)

[36] J. Weiland and H. Nordman, Phys. Fluids B5, 1669 (1993)

[37] F. Jenko et al., Plasma Phys. Control. Fusion 47, B195 (2005)

[38] F. Jenko, Phys. Plasmas 7, 514 (2000)

[39] C. Angioni and A. G. Peeters, Phys. Rev. Lett. 96, 095003 (2006)

[40] C. Angioni et al., Phys. Plasmas 16, 060702 (2009)

[41] B. D. Scott, Plasma Phys. Control. Fusion 49, S25 (2007)

[42] M. Kotschenreuther et al., Comput. Phys. Commun. 88, 128 (1995)

[43] W. Dorland et al., Phys. Rev. Lett. 85, 5579 (2000)

[44] R. E. Waltz et al., Phys. Plasmas 2, 2408 (1997)

[45] M. Maslov et al., Iaea FEC 2008 Preprints EX/P5-20 (2008)

[46] F. Jenko, private communication

[47] J. Candy and R. E. Waltz, Journal Phys. Commun. 186, 545 (2003)

[48] H. Weisen et al., Plasma Phys. Control. Fusion 48, A457 (2006)

[49] C. Angioni et al., Nucl. Fusion 47, 1326 (2007)

[50] E. Fable et al., Plasma Phys. Control. Fusion 50, 115005 (2008)

[51] F. Ryter et al., Plasma Phys. Control. Fusion 48, B453 (2006)

[52] G. D. Conway et al., Nucl. Fusion 46, S799 (2006) 

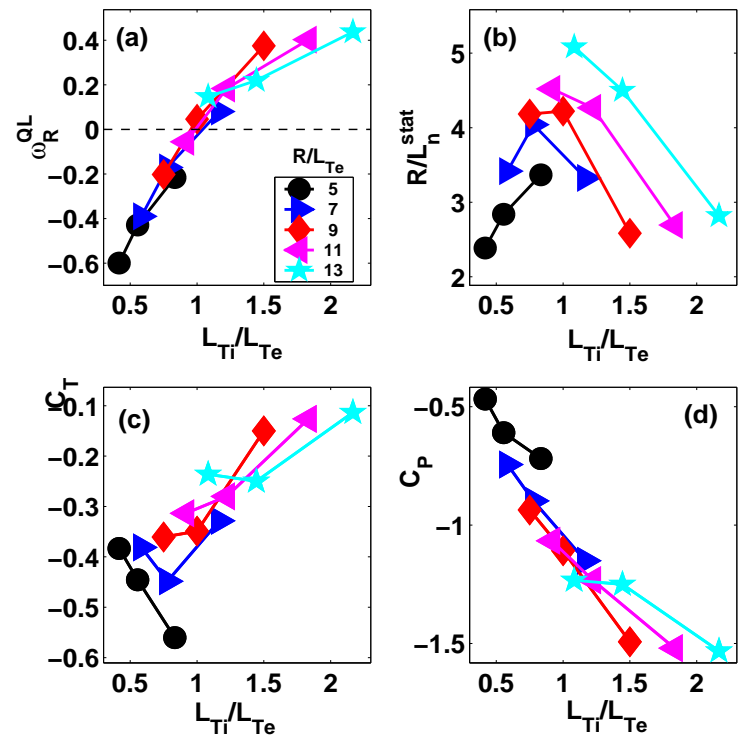

Figure 1. a) Averaged frequency $\omega_{\mathrm{R}}^{\mathrm{QL}}$; b) $\left[R / L_{\mathrm{n}}\right]_{\text {stat }}$; c) Thermodiffusion coefficient $C_{\mathrm{T}}$; d) The other pinch coefficient $C_{\mathrm{P}}$. All quantities are plotted versus $L_{\mathrm{Ti}} / L_{\mathrm{Te}}$ at different values of $R / L_{\mathrm{Te}}$ (legend). 

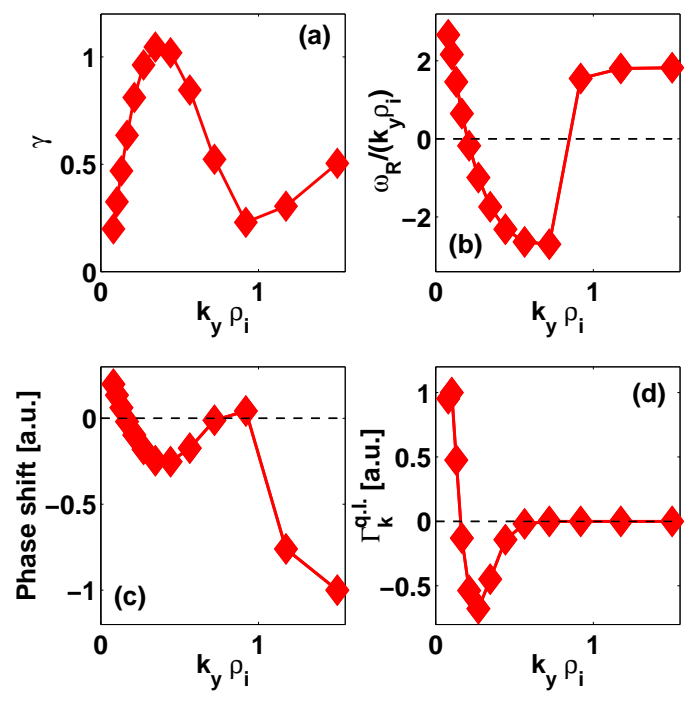

Figure 2. Spectrum, at stationary state, for the case $R / L_{\mathrm{Te}}=R / L_{\mathrm{Ti}}=9$ of figure 1 (diamonds), of: (a) the growth rate $\gamma$, (b) 'phase velocity' $\omega_{\mathrm{R}} /\left(k_{\mathrm{y}} \rho_{\mathrm{i}}\right),(\mathrm{c})$ phase shift, (d) quasi-linear flux. 


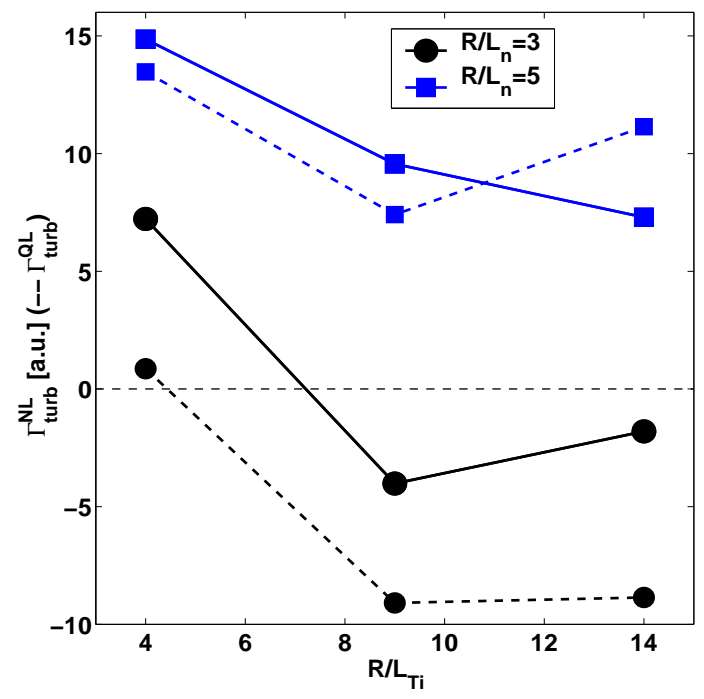

Figure 3. Non-linear particle flux in arbitrary units (solid lines) calculated with GYRO versus $R / L_{\mathrm{Ti}}$ for two values of $R / L_{\mathrm{n}}$ (legend) for the base case. The quasilinear flux (dashed lines) is plotted as well for comparison. The latter is obtained fixing the constat $A_{0}$ of equation (19) to match the average value between the square symbols. 

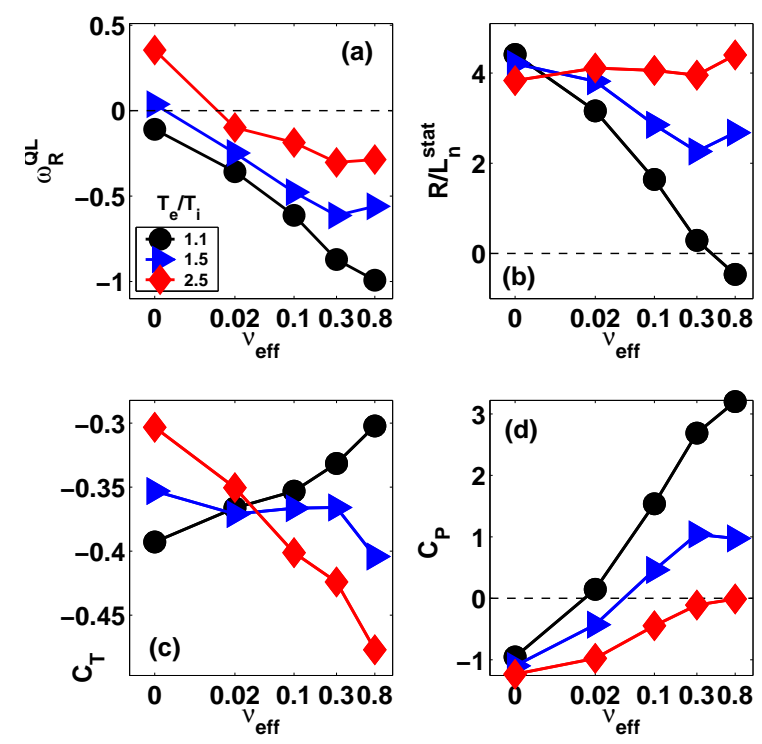

Figure 4. Same plots as in figure 1 , here for the $\hat{\nu}-T_{\mathrm{e}} / T_{\mathrm{i}}$ scan. We plot the curves versus $\hat{\nu}$ at different values of $T_{\mathrm{e}} / T_{\mathrm{i}}$ (legend). 

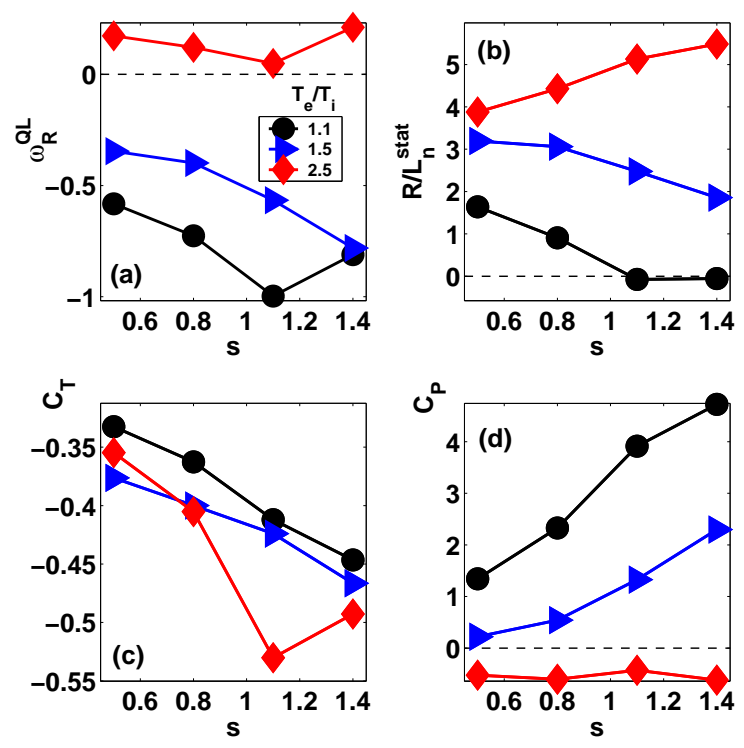

Figure 5. Same plots as in figure 1, here for the $s-T_{\mathrm{e}} / T_{\mathrm{i}}$ scan. We plot the curves versus $s$ at different values of $T_{\mathrm{e}} / T_{\mathrm{i}}$ (legend). 

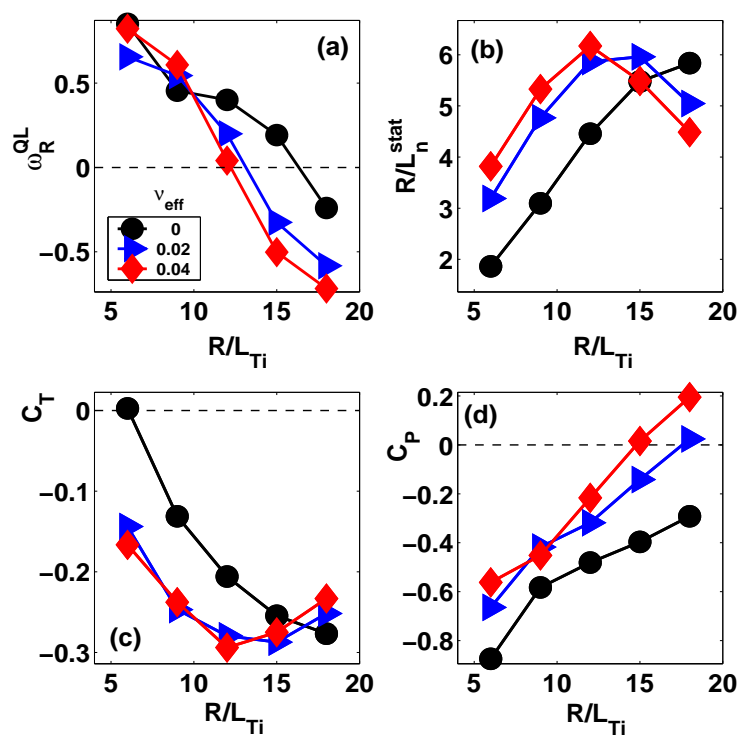

Figure 6. Same plots as in figure 1 , here for the $R / L_{\mathrm{Ti}}-\hat{\nu}$ scan. We plot the curves versus $R / L_{\mathrm{Ti}}$ at different values of $\hat{\nu}$ (legend). 

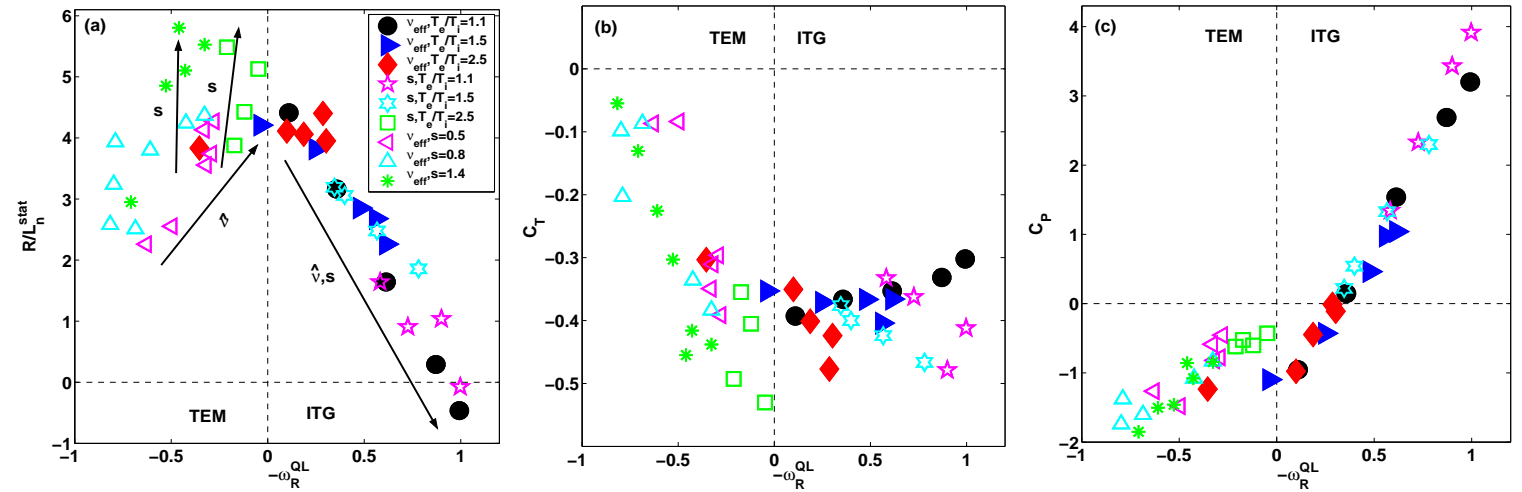

Figure 7. a) $\left[R / L_{\mathrm{n}}\right]_{\text {stat }}$ plotted versus the average frequency $\omega_{\mathrm{R}}^{\mathrm{QL}}$ for several parameters scan (in the legend); b) Thermodiffusion coefficient $C_{\mathrm{T}}$ for the same scans; c) The other pinch coefficient $C_{\mathrm{P}}$. 